\title{
Hétérosis et hérédité de quatre caractères agronomiques dans les croisements de lignées fixées de betterave fourragère et sucrière (Beta vulgaris $L$ )
}

\author{
F Le Cochec, P Soreau \\ INRA, centre de recherches de Rennes, station d'amélioration des plantes, domaine de la Motte au Vicomte,
} BP 29, 35650 Le Rheu, France

(Reçu le 24 mai 1991; accepté le 25 novembre 1991)

\begin{abstract}
Résumé - Les parents et les générations $F_{1}, F_{2}, B_{1}, B_{2}$, issues du croisement d'une lignée fixée $P_{1}$ de betterave fourragère à faible teneur en matière sèche et d'une lignée $P_{2}$ de betterave sucrière ont été testés dans 2 croisements différents pour les caractères poids frais des racines, teneur en matière sèche et poids sec des racines et dans un croisement pour le caractère de coloration des racines.

Les variations observées pour les caractères poids frais et poids sec des racines dans les différentes générations ont été expliquées principalement par des effets de dominance et d'épistasie digénique. Toutefois, dans un croisement, les variations du poids frais des racines ne peuvent s'expliquer qu'en faisant intervenir des effets d'épistasie trigénique (ou d'un ordre supérieur) et de linkage entre loci. Pour le caractère teneur en matière sèche, il y a prédominance des effets d'additivité avec, néanmoins, dans chaque croisement, des effets de dominance et d'épistasie très significatifs. Ce caractère apparaît conditionné par au moins 5 paires de gènes.

Les manifestations d'hétérosis, très significatives, sont dues à des effets de dominance, de superdominance et d'épistasie pour les 3 caractères dans les 2 croisements. Les taux d'héritabilité au sens large sont bas ou très bas $(0,25-$ $0,49)$ pour les poids frais et sec des racines et élevés $(0,62-0,89)$ pour la teneur en matière sèche. Les corrélations du poids frais et de la teneur en matière sèche sont négatives et généralement prononcées. Les relations entre le poids sec et la teneur en matière sèche sont faibles et inconsistantes. La coloration des racines est déterminée par des allèles présents à 2 loci non liés $R$ et $Y$.

Les résultats de cette étude mettent en évidence l'importance, la fréquence et la diversité des effets d'épistasie dans l'expression des caractères quantitatifs étudiés. L'hétérosis apparaît, pour l'essentiel, comme le résultat d'effets d'épistasie d'ordre élevé au stade juvénile.
\end{abstract}

Beta vulgaris = betterave fourragère / Beta vulgaris = betterave sucrière / poids des racines / teneur en matière sèche / couleur des racines / hétérosis / hérédité

Summary - Heterosis and inheritance of four agronomic characters in sugar beet and mangel (Beta vulgaris L) inbred line crosses. Parents, $F_{1}, F_{2}$ and backcross generations of 2 crosses between sugar beet and mangel inbred lines were evaluated for root weight, percentage dry matter and dry matter yield characters. The root colour character was observed in one cross. Variation among generation means for root weight and dry matter yield characters were mainly explained by dominance and digenic epistatic effects. However, in one cross, variation for root weight character could not be explained without trigenic (or higher order) interactions and linkage between the pairs of loci. For the character percentage dry matter, additivity effects were predominant with very significant dominance and epistatic effects. The minimum number of pairs of genes affecting this character was 4 in one cross and 5 in the other cross (tables I, II, III). Phenotypic dominance and significant heterosis due to dominance, overdominance and epistasis occurred in the 2 crosses and the 3 characters. Broad sense heritability estimates were low (0.25-0.49) for the root weight and dry matter yield and high $(0.62-0.89)$ for dry matter percent (table IV). The correlations for weight of roots and dry matter percent were negative and frequently pronounced. Correlations for dry matter yield and dry matter percent were weak and inconsistent (table $V$ ). Root colour was determined by alleles at two loci, $R$ and $Y$, and these two loci were not linked (table VI). The results showed the importance, frequency and diversity of epistatic effects in the expression of the quantitative characters under study. Heterosis resulted primarily from high order epistatic effects at a young stage.

Beta vulgaris = sugar beet $/$ Beta vulgaris = mangel $/$ root weight $/$ dry matter percent $/$ dry matter yield $/$ root colour / heterosis / inheritance 


\section{INTRODUCTION}

Les variétés monogermes de betterave fourragère ont été cultivées, en 1989, en France, sur 37500 ha et dans l'ensemble des pays de la CEE, la même année, sur 230000 ha. Ce sont des variétés hybrides obtenues généralement par le croisement d'un parent monogerme mâlestérile de betterave sucrière et d'un parent pollinisateur, diploïde ou tétraploïde, de betterave fourragère à faible teneur en matière sèche.

Lors des épreuves d'inscription aux catalogues officiels des différents pays de la Communauté, la valeur agronomique et technologique de ces variétés est estimée, non pas à partir du rendement et de la teneur en sucre comme c'est le cas pour les variétés de betterave sucrière, mais à partir du rendement en matière sèche par ha et de la teneur en matière sèche des racines. D'après Demarquilly (1972), la matière sèche des racines de betterave fourragère est constituée par 64 à $66 \%$ de sucres (essentiellement du saccharose) et comporte en outre des pectines, des celluloses et hémicelluloses, des protéines, des matières grasses (en faible quantité) et des matières minérales.

Plusieurs travaux, analysés ci-dessous, ont été consacrés à l'étude de l'hérédité et du mode d'action des gènes qui conditionnent le poids des racines, la teneur en sucre et le rendement en sucre des variétés de betterave sucrière. Cependant, aucune publication, à notre connaissance du moins, n'a concerné jusqu'à présent l'étude des caractères propres aux variétés de betterave fourragère : poids des racines, teneur en matière sèche et rendement en matière sèche des racines. Une connaissance plus précise de l'hérédité de ces 3 caractères est pourtant d'un grand intérêt car elle permettrait de mieux définir l'importance et la nature de l'hétérosis et donc une stratégie raisonnée de l'amélioration de ces variétés.

Les objectifs de cette étude ont consisté à analyser, pour les 3 caractères, les moyennes et les variances de 6 générations issues de 2 croisements entre lignées différentes de betterave sucrière et fourragère à faible teneur en matière sèche. Il s'agit de lignées fixées diploïdes, choisies au hasard dans une population de lignées. Cette analyse a permis de préciser le mode d'action des gènes, la nature de l'hétérosis, le taux d'héritabilité et les relations entre caractères. L'hérédité de la coloration des racines $a$, en outre, été analysée dans l'un des 2 croisements.

\section{MATÉRIEL ET MÉTHODES}

Les croisements I et II ont été réalisés entre les lignées suivantes:

\section{Croisement I : OLI $\left(P_{1}\right) \times 561-20\left(P_{2}\right)$}

OLI est une lignée pseudocompatible de betterave fourragère à hypocotyle vert et racine orange, autofécondée pendant 9 générations consécutives. Elle provient de la variété belge Olivat.

561-20 est une lignée autofertile de betterave sucrière à hypocotyle rouge. II s'agit d'un mainteneur de stérilité (type 0) monogerme, sélectionné dans la souche USDA : C 9561 (McFarlane et Skoyen, 1965). Cette lignée a été maintenue en autofécondation pendant 8 générations consécutives et recroisée chaque année sur le parent mâle-stérile correspondant : C 9561 HO 20. Type 0 et lignée mâle-stérile ont un aspect et des caractères identiques.

\section{Croisement II : RVE $\left(P_{1}\right) \times 569-31\left(P_{2}\right)$}

RVE est une lignée pseudocompatible de betterave fourragère à hypocotyle vert et racine blanche, autofécondée pendant 10 générations consécutives. Elle provient de la variété de betterave fourragère française, maintenant disparue, Reine des Vertes, proche de Géante Blanche.

569-31 est une lignée autofertile de betterave sucrière qui possède les mêmes caractéristiques que la lignée 561-20. Elle provient de la souche USDA: C-7569.

Ces 4 lignées représentent les extrêmes pour la teneur en matière sèche des variétés actuelles de betterave fourragère et sucrière.

Les parents $P_{1}$ et $P_{2}$ et les générations $F_{1}$, mâlestérile et mâle-fertile, $F_{2}$ issue de la multiplication de la génération mâle-fertile $F_{1}, B_{1}$ et $B_{2}$ provenant respectivement du croisement des $F_{1}$ mâle-stériles par les parents $P_{1}$ et $P_{2}$ ont été utilisés dans cette expérimentation.

Les plantes $F_{1}$ mâle-fertiles, récoltées sur le parer.t $P_{1}$, ont été identifiées à l'aide du gène marqueur dominant $\mathbf{R}$ qui colore l'hypocotyle en rouge. Les lignées OLl et RVE se comportent comme un type 0 en croisement avec les lignées mâle-stériles de sorte que l'hybride mâle-stérile a pu être utilisé pour obtenir les générations $B_{1}$ et $B_{2}$. Dans un premier temps un certain nombre de plantes $F_{1}$ mâle-fertiles (destinées à la production de la génération $F_{2}$ ) et $F_{1}$ mâle-stériles (destinées à la production des générations $B_{1}$ et $B_{2}$ ) ont été obtenues sous des isoloirs de popeline. L'année suivante, les 6 générations $P_{1}, P_{2}, F_{1}$ (mâlestérile), $F_{2}, B_{1}$ et $B_{2}$ ont été récoltées dans des isolements par la distance.

Au cours de la troisième année, ces 6 générations ont été semées en lignes continues dans un essai en blocs randomisés à 8 répétitions. Le niveau de fertilisation des essais a été élevé en $P$ et $K$ et très élevé en $\mathrm{N}$. Les traitements herbicides, aphicides et fongi- 
cides recommandés par l'Institut Technique de la Betterave ont été appliqués aux époques conseillées. Chaque parcelle d'essai comportait 5 lignes de $5,50 \mathrm{~m}$ (croisement I) ou $6,50 \mathrm{~m}$ (croisement II) espacées de $0,50 \mathrm{~m}$. Le démariage a été effectué très soigneusement, à raison d'une plante tous les $0,25 \mathrm{~m}$. Seules les 3 lignes centrales de chaque parcelle ont été récoltées, à l'exception toutefois des 2 premières et des 2 dernières plantes de chaque ligne, soit un nombre théorique de 19 ou 23 betteraves récoltées par ligne. Seules les racines en situation de compétition normale ont été récoltées (Cette sujétion explique qu'une orthogonalité parfaite des données n'a pu être obtenue dans les 2 croisements).

Le croisement I a été semé le 26 avril 1988, démarié le 9 juin, récolté et analysé entre le 29 septembre et le 26 octobre à raison d'une répétition par jour de récolte.

Le croisement II a été semé le 26 avril 1990, démarié le 23 mai, récolté et analysé entre le 10 et le 29 octobre, dans les mêmes conditions que le croisement I.

Les racines ont été effeuillées à la base des pétioles mais non décolletées, comme il est d'usage pour les betteraves fourragères. Elles ont ensuite été lavées dans une parmentière tournant à faible vitesse, séchées à l'air libre et pesées à $1 \mathrm{~g}$ près.

La matière sèche a été déterminée sur un secteur prélevé longitudinalement dans chaque racine et pesant entre 56 et $139 \mathrm{~g}$ suivant la taille de la racine. Ce secteur a été aussitôt pesé, découpé en cossettes de $3 \mathrm{~mm}$ d'épaisseur et desséché par passage durant 48 $h$ dans une étuve ventilée réglée à $80^{\circ} \mathrm{C}$. Les poids frais et sec de l'échantillon ont été estimés au cg près. Le poids sec a ensuite été calculé pour chaque racine.

Les rendements en matière sèche des racines correspondent à des rendements moyens mais les teneurs en matière sèche ont été, les 2 années, supérieures à la normale.

La coloration blanche, orange ou rouge des racines a été notée pour chacune des générations du croisement I.

\section{Méthodes statistiques}

L'analyse de variance pour les 3 caractères : poids frais, poids sec des racines et teneur en matière sèche montre que l'effet des répétitions est significatif ou très significatif. La variabilité due aux différences entre les moyennes des répétitions a été annulée en ajoutant ou en retranchant une constante aux individus de chaque parcelle à l'intérieur de chaque répétition. Cet ajustement ne modifie pas la moyenne de chaque génération mais il a permis une réduction notable des variances intraparcellaires par rapport aux données non ajustées, pour le caractère teneur en matière sèche en particulier. Tous les calculs ont ensuite été effectués sur les données ainsi ajustées.

\section{Test de normalité des distributions}

Les fréquences de distribution obtenues ont été comparées aux fréquences de distribution d'une courbe normale à l'aide d'un test $\chi^{2}$. Dans les faibles fréquences, plusieurs classes ont été regroupées de façon à obtenir une valeur théorique au moins égale à 1. Pour les valeurs de $\chi^{2}$ correspondant à une probabilité inférieure à 0,05 d'être dépassées, on a considéré que la courbe de distribution n'était pas normale.

\section{Analyse des moyennes}

Après estimation des écarts types respectifs, les moyennes observées de chaque génération ont été comparées, dans un premier temps, aux moyennes arithmétiques (tous les effets des gènes s'additionnent, il n'y a pas d'effets d'interaction intra- ou interallélique).

Elles ont ensuite été utilisées pour estimer les composantes de la moyenne, le mode d'action des gènes et les valeurs d'hétérosis à l'aide des méthodes décrites par Mather et Jinks (1977) et Jinks (1983). Les valeurs moyennes de chaque génération ont été comparées aux valeurs attendues, obtenues dans le modèle à 3 paramètres (moyenne $m$, additivité $d$, dominance $h$ ) à l'aide d'un test $\chi^{2}$. Les valeurs de $\chi^{2}$ ont été calculées en pondérant les écarts entre les valeurs observées et théoriques par l'inverse de leurs variances. Les valeurs de ce test étant toutes très significatives pour les 3 caractères, les moyennes observées ont été utilisées pour calculer en outre les 3 effets d'épistasie digénique : $i$ : additivité $\mathrm{x}$ additivité, $j$ : additivité $\mathrm{x}$ dominance, $l$ : dominance $\mathrm{x}$ dominance. Les 6 moyennes observées permettent une estimation exacte des 6 paramètres ainsi définis ainsi que le calcul d'un test de signification de la valeur de ces paramètres. Les paramètres significativement différents de zéro ainsi que leurs écarts types ont été calculés de la même manière que pour le modèle à 3 paramètres précédent et l'adéquation du modèle aux données a pu être testée également à l'aide d'un $\chi^{2}$.

Les valeurs d'hétérosis ont été estimées, soit par la déviation de la $F_{1}$ par rapport à la moyenne des parents :

- en valeur absolue : $F_{1}-1 / 2\left(P_{1}+P_{2}\right)$, ou

- en valeur relative : $F_{1}-1 / 2\left(P_{1}+P_{2}\right) / 1 / 2\left(P_{1}+P_{2}\right)$,

soit par la mesure de l'effet de superdominance. Elles ont aussi été calculées (sauf dans un cas de valeur très significative du test $\chi^{2}$ ) à partir des paramètres génétiques composantes de la moyenne.

Les moyennes géométriques (antilogarithmes des moyennes obtenues après transformation logarithmique des moyennes observées) et la valeur des potences (Mather et Jinks, 1977) : 


$$
\left|F_{1}-1 / 2\left(P_{1}+P_{2}\right)\right| /\left|1 / 2\left(P_{1}-P_{2}\right)\right|
$$

ont également été estimées dans l'analyse des moyennes.

\section{Analyse des variances}

II n'y a pas, dans les 3 caractères, proportionnalité entre les moyennes et les variances de la moyenne pour les générations $P_{1}, P_{2}$ et $F_{1}$ et il n'a donc pas semblé justifié de procéder à une transformation préalabe des données.

Pour chaque génération la variance intraparcellaire a été décomposée en une variance génétique et une variance environnementale.

Cette dernière variance a été estimée de la façon suivante. Comme il n'y a, pour aucun caractère des 2 croisements, homogénéité des variances intraparcellaires dans les 3 générations $P_{1}, P_{2}$ et $F_{1}$, la moyenne de ces 3 variances n'a pu être utilisée pour estimer la variance environnementale. Cette variance a été calculée en utilisant une régression linéaire des variances intraparcellaires sur les moyennes. Cette régression rend compte entre 91,7 et $99,9 \%$ de la variation observée dans les 2 croisements. Toutefois, dans le croisement I, la distribution de la teneur en matière sèche n'étant pas normale pour la génération $F_{1}$, seules les générations $P_{1}$ et $P_{2}$ ont été utilisées dans le calcul de la régression. Dans le croisement II, pour le même caractère, une régression quadratique a mieux expliqué la variation observée qu'une régression linéaire $(99,8 \%$ de la variation au lieu de $66,0 \%)$. Les coefficients de la régression sont tous, (sauf pour la génération $F_{1}$ du croisement $l$ ), très différents de zéro.

Les variances génétiques ont été obtenues par différence des variances intraparcellaires et des variances environnementales ainsi calculées et leur niveau de signification a été déterminé à l'aide d'un test $F$. L'héritabilité du caractère, au sens large, a ensuite été calculée dans les générations en ségrégation.

\section{Relations entre caractères}

Pour chaque couple de caractères, on a déterminé les covariances environnementales par des régressions linéaires dans les générations non en ségrégation suivant la méthode indiquée par Pylman (1968). Les corrélations entre les covariances phénotypiques et les moyennes, comprises entre 0,81 et 0,99 , sont très significatives et indiquent que ces régressions permettent une bonne estimation des covariances environnementales. Les covariances génétiques sont obtenues par différence. Les corrélations génétiques sont le rapport des covariances génétiques au produit des écarts types génétiques des 2 caractères.

\section{RÉSULTATS}

\section{Normalité des distributions de fréquences}

La valeur et la probabilité des tests $\chi^{2}$ de comparaison des fréquences de distribution observées et calculées pour une courbe normale ont été indiquées, pour chaque génération, sur le tableau I.

Les fréquences de distribution des générations non en ségrégation ne sont pas différentes de celles d'une courbe normale, à l'exception, toutefois, de la teneur en matière sèche de la génération $\mathrm{F}_{1}$ du croisement I. La forte asymétrie à droite de cette distribution, qui se retrouve dans plusieurs répétitions, n'a pu être expliquée. En effet, toutes les plantes de cette génération présentaient une très grande homogénéité phénotypique (les hybrides accidentels auraient donc été rapidement identifiés) de sorte que les déviations constatées ne peuvent être que l'effet du milieu (présence de jaunisse tardive peut-être) et du hasard. II a été tenu compte de cette situation dans les calculs ci-dessous.

Dans les générations en ségrégation, les fréquences de distribution ne sont généralement pas distribuées suivant une courbe normale (à l'exception, fréquente, des générations du caractère teneur en matière sèche). Ceci peut être une conséquence, soit de la variabilité génétique, soit de la variabilité due au milieu, soit des deux à la fois. Les chiffres correspondant à quelques-unes de ces distributions non normales ont subi les transformations logarithmique ou racine carrée sans qu'il en résulte toujours une amélioration significative de l'adaptation à une courbe normale. En définitive, nous avons préféré analyser les résultats obtenus sur les chiffres ajustés effectivement observés.

\section{Analyse des moyennes}

Les moyennes observées et les moyennes arithmétiques ainsi que leurs écarts types sont indiqués, pour chaque génération, sur le tableau II.

Pour le poids frais des racines les différences entre les moyennes des générations sont toutes (à l'exception de 3 cas) très significatives.

La moitié de l'effet d'hétérosis est perdu par le passage de la génération $F_{1}$ à la génération $F_{2}$ et les $F_{1}$ observées sont beaucoup plus élevées 
Tableau I. Comparaison des fréquences de distribution observées et calculées, test $\chi^{2}$ et probabilités correspondantes pour le poids frais, la teneur en matière sèche et le poids sec des racines dans les 6 générations.

\begin{tabular}{|c|c|c|c|c|c|c|c|c|c|c|}
\hline \multirow[t]{2}{*}{ Génération } & \multirow[t]{2}{*}{ Nombre de racines } & \multicolumn{3}{|c|}{ Poids frais } & \multicolumn{3}{|c|}{ Teneur en matière sèche } & \multicolumn{3}{|c|}{ Poids sec } \\
\hline & & $d d l$ & $\chi^{2}$ & $P$ & $d d l$ & $\chi^{2}$ & $\mathbf{P}$ & $d d l$ & $\chi^{2}$ & $\mathbf{P}$ \\
\hline
\end{tabular}

Croisement I : $O L I\left(P_{1}\right) \times 561-20\left(P_{2}\right)$

$\begin{array}{lrrrrrrrrrr}\mathrm{P}_{1} & 398 & 9 & 12,3 & 0,25-0,50 & 6 & 9,1 & 0,10-0,25 & 9 & 13,2 & 0,10-0,25 \\ \mathrm{P}_{2} & 398 & 11 & 14,3 & 0,10-0,25 & 6 & 5,3 & 0,50-0,75 & 8 & 11,9 & 0,10-0,25 \\ \mathrm{~F}_{1} & 414 & 10 & 13,4 & 0,10-0,25 & 8 & 38,1 & 0,01 & 16 & 17,4 & 0,25-0,50 \\ \mathrm{~F}_{2} & 402 & 12 & 26,0 & 0,01-0,02 & 23 & 37,8 & 0,02-0,05 & 15 & 31,4 & 0,01 \\ \mathrm{~B}_{1} & 367 & 15 & 38,9 & 0,01 & 12 & 15,3 & 0,10-0,25 & 19 & 39,4 & 0,01 \\ \mathrm{~B}_{2} & 391 & 11 & 35,0 & 0,01 & 13 & 8,9 & 0,75-0,90 & 16 & 41,7 & 0,01\end{array}$

Croisement II : RVE $\left(P_{1}\right) \times 569-31\left(P_{2}\right)$

$\begin{array}{llrrlrrrrrr}\mathrm{P}_{1} & 500 & 16 & 14,2 & 0,50-0,75 & 11 & \mathbf{8 , 1} & 0,50-0,75 & 15 & 14,8 & 0,25-0,50 \\ \mathrm{P}_{2} & 509 & 9 & 5,1 & 0,75-0,90 & 7 & 9,4 & 0,10-0,25 & 11 & 10,1 & 0,50-0,75 \\ \mathrm{~F}_{1} & 541 & 16 & 14,1 & 0,50-0,75 & 9 & 15,5 & 0,05-0,10 & 13 & 17,9 & 0,10-0,25 \\ \mathrm{~F}_{2} & 537 & 16 & 42,2 & 0,01 & 12 & \mathbf{8 , 9} & 0,50-0,75 & 12 & 25,3 & 0,01-0,02 \\ \mathrm{~B}_{1} & 510 & 16 & 29,2 & 0,01 & 10 & 15,8 & 0,10-0,25 & 12 & 22,2 & 0,02-0,05 \\ \mathrm{~B}_{2} & 516 & 12 & 62,1 & 0,01 & \mathbf{8} & \mathbf{6 , 0} & 0,50-0,75 & 12 & 63,0 & 0,01\end{array}$

que les $F_{1}$ arithmétiques. II existe souvent des différences très importantes entre les valeurs observées et les valeurs arithmétiques des générations en ségrégation. Ces observations constituent une présomption de la faible importance des gènes à effet additif et de l'importance des effets de dominance et d'épistasie.

Dans le croisement I, les moyennes des générations $F_{1}$ et $B_{1}$ sont égales, ce qui indique que la totalité des gènes à effet dominant provient du parent $P_{1}$.

Dans le caractère pourcentage de matière sèche, les moyennes observées sont toutes différentes entre elles à l'exception des générations $F_{1}$ et $F_{2}$.

Leur examen montre que l'action additive des gènes est prépondérante. Toutefois, dans les 2 croisements, la valeur observée de la $F_{1}$ est très significativement supérieure à la moyenne arithmétique, ce qui traduit une dominance phénotypique des teneurs en matière sèche élevées sur les basses teneurs. La faible valeur de la potence $(0,05$ et 0,15$)$ indique cependant que cette dominance est peu importante et cela est confirmé par l'égalité des moyennes $F_{1}$ et $F_{2}$ et le fait que les moyennes observées sont beaucoup plus proches des moyennes arithmétiques que des moyennes géométriques.

La présence d'effets d'épistasie est déduite des différences significatives (ou très significa- tives) entre les moyennes observées et arithmétiques dans la plupart des générations en ségrégation.

Le poids sec des 2 parents du croisement I est le même et on peut donc penser que l'hétérosis, représenté par la différence entre les moyennes observée et arithmétique de la $F_{1}$, est due en totalité à des effets de dominance et d'épistasie.

Dans les 2 croisements, la présence des effets d'épistasie est une conséquence des différences significatives existant entre les valeurs observées et calculées des 3 générations en ségrégation. Les potences pour le poids sec atteignent des valeurs très élevées dans les 2 cas. Les moyennes des générations $B_{1}$ et $B_{2}$ étant sensiblement égales, on peut penser que les gènes qui conditionnent le rendement en matière sèche des racines proviennent en fréquence égale des 2 parents.

\section{Mode d'action des gènes et hétérosis}

Les composantes de la moyenne, l'adaptation des différents modèles aux moyennes observées ainsi que les valeurs d'hétérosis pour les 3 caractères sont indiquées sur le tableau III.

Les éléments de ce tableau apportent des précisions à l'examen direct des moyennes ob- 


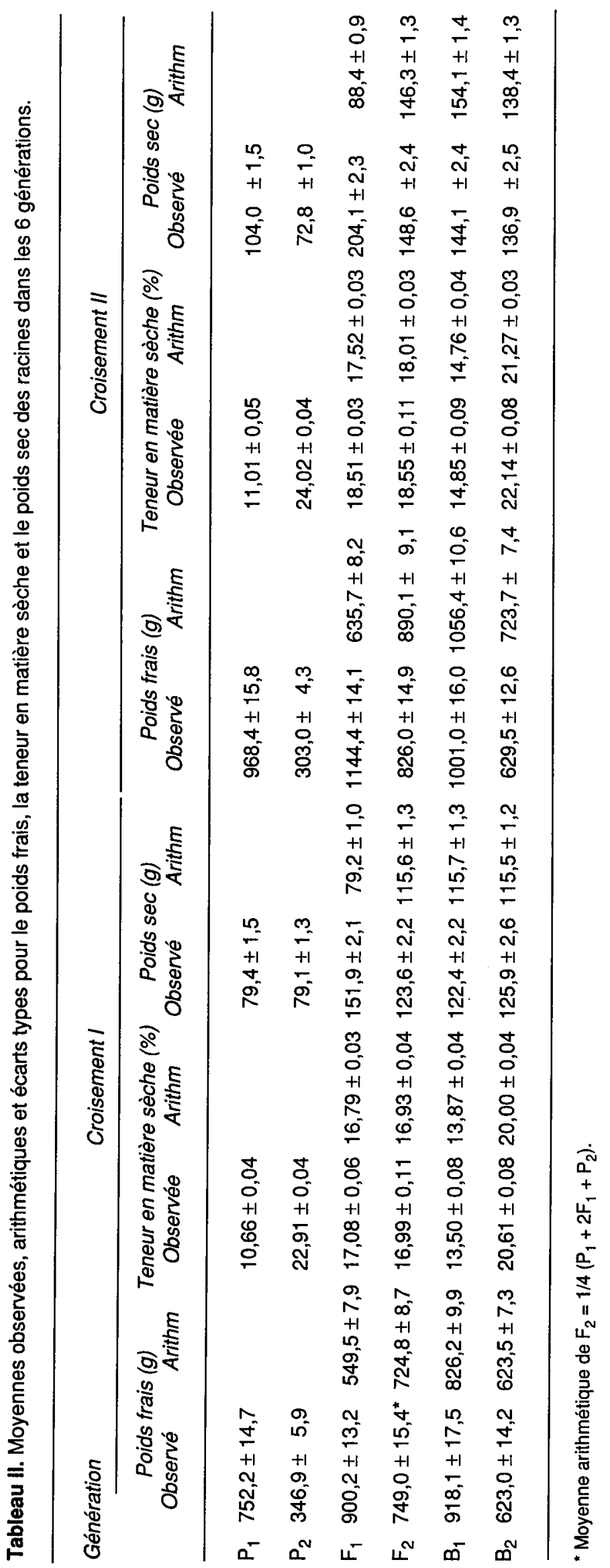


Tableau III. Composantes de la moyenne, adaptation des modèles et hétérosis pour le poids frais, la teneur en matière sèche et le poids sec des racines dans les 6 générations.

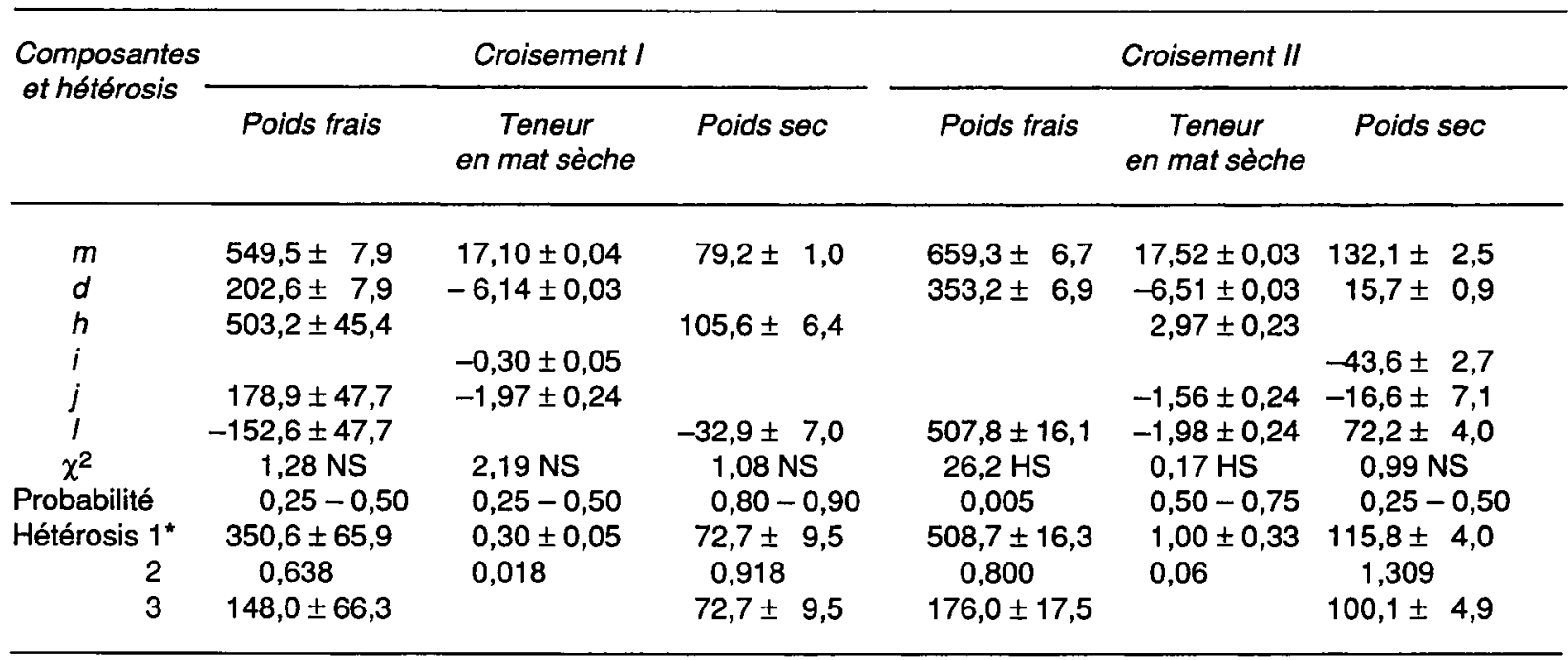

* 1 - Hétérosis : $h+1-i ; 2-$ Hétérosis relatif : $h+l-i / m+i ; 3-$ Superdominance $: h+1-d-i$.

servées. Ces moyennes sont expliquées (à l'exception d'un seul cas) par des modèles à 3,4 ou 5 paramètres génétiques faisant intervenir des effets d'additivité, de dominance et d'épistasie.

Les caractères poids frais et poids sec des racines sont dus, dans le croisement I, pour l'essentiel, à des effets de dominance modifiés par des effets d'épistasie de type dupliqué ( $h$ et / sont de signe contraire). Dans le croisement II, au contraire, les effets de dominance sont nuls et les effets d'épistasie jouent un rôle essentiel dans l'expression de ces deux caractères. Toutefois, dans ce dernier croisement, le caractère poids frais des racines n'a pu être expliqué uniquement par des effets d'épistasie digénique (la valeur du $\chi^{2}$ est hautement significative). La cause la plus vraisemblable de l'incapacité du modèle comportant les paramètres $m, d$ et $/$ d'expliquer les moyennes observées est l'existence d'interactions trigéniques (ou d'un ordre supérieur) ou, peut-être, la présence de linkage entre loci. Cependant, la preuve formelle de la présence d'interactions de ce type aurait nécessité la réalisation des seconds backcrosses ce qui n'a pas été fait ici.

Le caractère teneur en matière sèche est bien, comme l'avait fait supposer l'analyse des moyennes, pour une grande part, une conséquence de l'action de gènes à effet additif mais cette action est profondément modifiée par des effets de dominance ou d'épistasie (ou les deux) très significatifs.

Les valeurs d'hétérosis (déviation de la F1 par rapport à la moyenne des parents) sont toutes très significativement différentes de zéro pour les
3 caractères. Les valeurs de superdominance sont significativement ou très significativement différentes de zéro pour le poids frais et le poids sec des racines. Il s'agit d'une conséquence de l'importance des effets de dominance et d'épistasie présents dans l'expression de ces caractères. L'importance de ces effets est sans doute liée elle-même à la distance génétique séparant les vieilles variétés européennes de betterave fourragère des variétés américaines de betterave sucrière.

\section{Analyse des variances}

Les variances intraparcellaires, environnementales et génétiques, ainsi que les valeurs de $\mathrm{F}$ et d'héritabilité au sens large ont été indiquées, pour chaque caractère et chaque génération, sur le tableau IV.

La génération $F_{1}$ du croisement I n'a pas été utilisée, comme on l'a dit, dans le calcul de la variance environnementale pour le caractère teneur en matière sèche.

La génération $P_{1}$ du croisement II semble présenter, assez inexplicablement, une variabilité génétique très proche du seuil de signification pour le poids frais des racines. L'existence d'une telle variabilité est peu probable, en raison du degré d'homozygotie très élevé de la lignée, et il est plus vraisemblable qu'il s'agit simplement d'une conséquence du mode de calcul de la variance environnementale. 


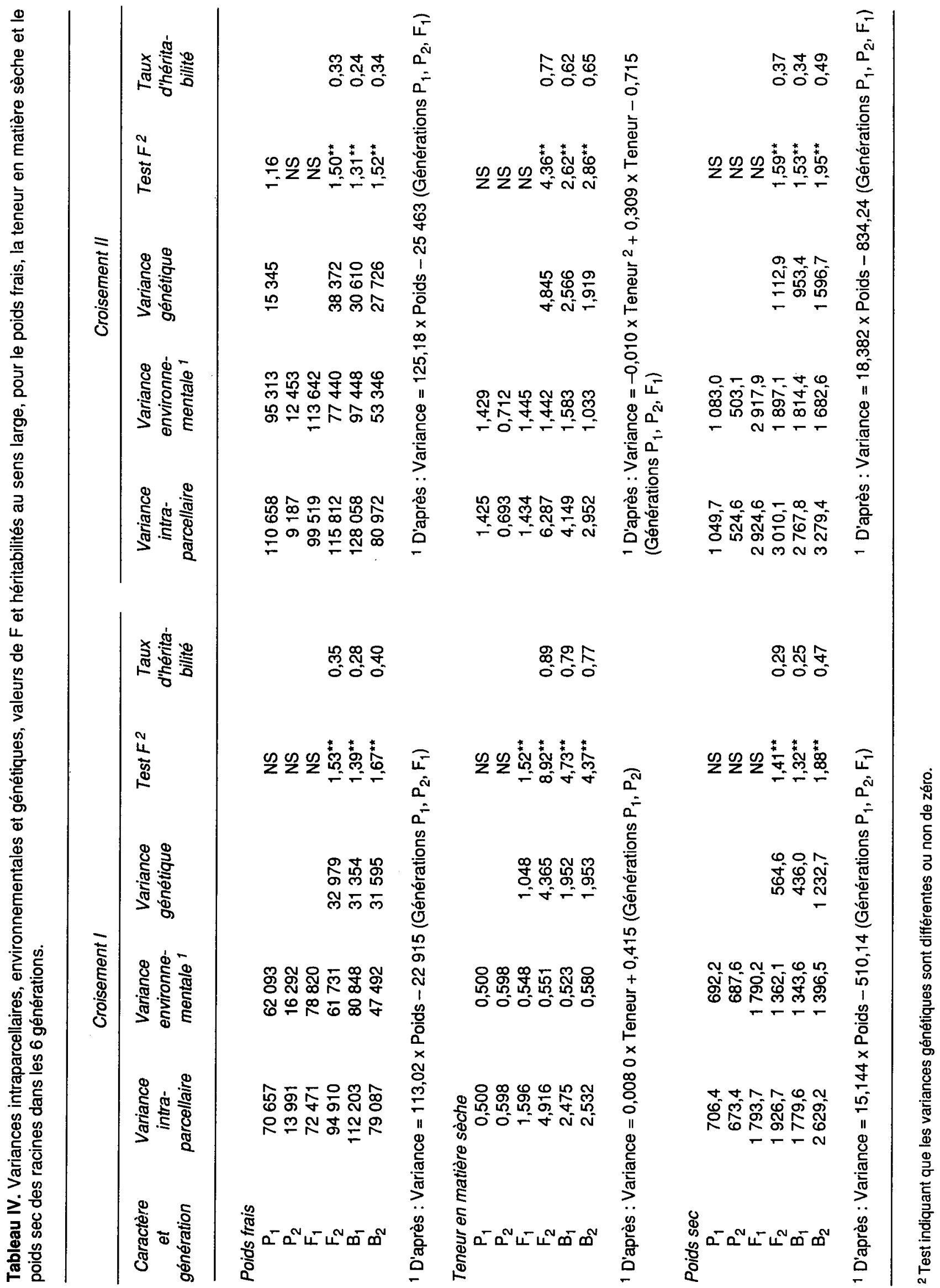


Les variances génétiques pour les 3 caractères sont toutes très significatives dans les générations en ségrégation.

Les héritabilités sont faibles à très faibles, et du même ordre pour les caractères poids frais $(0,24-0,40)$ et poids sec $(0,25-0,49)$ des racines. Les héritabilités élevées $(0,62-0,89)$ pour la teneur en matière sèche confirment l'importance du mode d'action additif des gènes pour ce caractère.

Les variances intraparcellaires et l'héritabilité de la génération $\mathrm{F} 2$ ont permis de calculer le gain relatif attendu en sélection pour un taux de sélection de $5 \%$. Ce gain est respectivement de 29,3 et $28,0 \%$ pour le poids frais, 23,9 et $21,5 \%$ pour la teneur en matière sèche, 12,9 et $28,1 \%$ pour le poids sec des racines.

\section{Relations entre caractères}

Les covariances et les corrélations phénotypiques et génétiques des 3 caractères pris 2 à 2 sont indiquées pour chaque génération sur le tableau $\mathrm{V}$.

Les corrélations phénotypiques sont toutes (sauf dans 2 cas) très significatives.

Les corrélations génétiques calculées sont la conséquence des effets d'additivité, de dominance et d'interaction des gènes entre eux. Elles ont été estimées avec une faible précision, en raison sans doute de l'erreur attachée au calcul de la covariance environnementale : certains coefficients de corrélation sont supérieurs à 1 et il existe une variation notable entre les générations en ségrégation d'un même croisement.

L'examen des corrélations génétiques indique néanmoins les tendances suivantes :

- il existe fréquemment une corrélation négative prononcée entre poids frais et teneur en matière sèche. Les corrélations environnementales sont également négatives et généralement comprises entre 0,40 et 0,50 . II y a donc une présomption pour que cette corrélation inverse entre les $2 \mathrm{ca}-$ ractères résulte de phénomènes de pléiotropie pour la plupart des gènes en cause;

- la liaison génétique entre poids frais et poids sec des racines est caractérisée par des corrélations génétiques et environnementales uniformément élevées et proches de l'unité. Les 2 caractères apparaissent, dans ces 2 croisements du moins, influencés par les mêmes gènes et on peut également privilégier l'hypothèse présence de pléiotropie. Les racines les plus volumineuses sont celles qui présentent le rendement en matière sèche le plus élevé;

- les corrélations génétiques et environnementales entre les caractères poids sec et pourcentage de matière sèche des racines sont, par contre, peu élevées et variables, tantôt positives, tantôt négatives. II est difficile de dire s'il existe bien une liaison entre ces 2 caractères et si cette liaison est due à des phénomènes de linkage ou de pléiotropie.

\section{Nombre de gènes différenciant les caractères}

La formule de Wright (1968), modifiée par Lande (1981) et Cockerham (1986), fait intervenir le carré de la différence entre les moyennes des générations parentales ainsi que la variance totale due aux différences de fréquences géniques dans ces 2 générations pour estimer le nombre des gènes affectant un caractère quantitatif.

L'application de cette formule nécessite toutefois la réalisation de plusieurs conditions préalables et, entre autres, que :

- les deux parents soient homozygotes, l'un ayant tous les gènes accroissant le caractère et l'autre tous les gènes le diminuant et que les populations utilisées $\left(P_{1}, P_{2}, F_{1}, F_{2}\right)$ soient distribuées normalement;

- les gènes en cause aient la même importance, qu'il n'y ait pas de linkage entre eux et que toute la variance génétique soit additive.

Le caractère teneur en matière sèche satisfait, apparemment, à la plupart de ces conditions. La méthode des moindres carrés non pondérée indique alors que le nombre de paires de facteurs conditionnant le caractère est de $5,5 \pm 0,4$ dans le croisement l et de 4,2 $\pm 0,3$ dans le croisement II soit, vraisemblablement, 5 et 4 paires de facteurs. Ce nombre est compatible avec l'hypothèse d'une absence de linkage entre gènes.

Cependant, la réalité et la précision des estimées fournies par la formule de Wright (modifiée ou non) ont été mises récemment en doute (Zeng et al, 1990). II est, en effet, très difficile d'affirmer que toutes les conditions préalables nécessaires à l'application de la formule ont bien été satisfaites. Dans ces conditions ce nombre de 4 ou de 5 paires de facteurs doit être considéré comme un nombre minimum. 

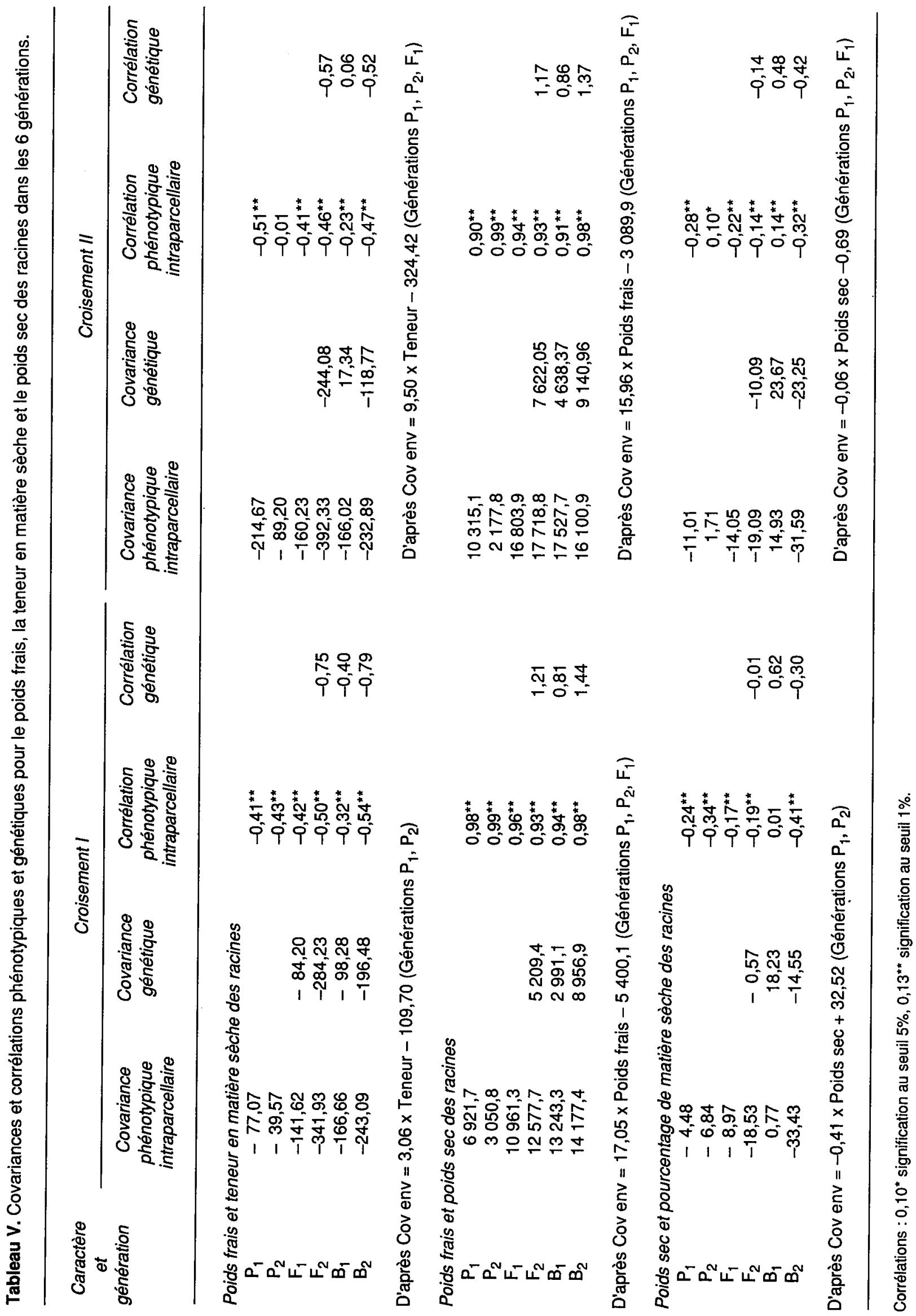


\section{Hérédité de la coloration des racines}

Kajanus (1917) a montré que la coloration des racines de betteraves fourragères et sucrières est déterminée par les allèles à 2 loci non liés, $R$ et $Y$. Au premier locus, le gène dominant $R$ conditionne la couleur rouge de l'hypocotyle et la couleur blanche des racines et le gène récessif $r$ la couleur verte de l'hypocotyle et la couleur blanche des racines. Au second locus, l'allèle dominant $Y$ provoque une coloration orange (ou jaune) et l'allèle récessif y une coloration blanche des racines. La coloration rouge est une conséquence de la présence d'allèles dominants aux 2 loci. Ces conclusions ont été confirmées ensuite par Lindhard et Iversen (1920).

Des études ultérieures de croisements de betteraves sucrières, fourragères et potagères ont établi l'existence de 2 séries alléliques aux loci $R$ et $Y$ et, en outre, la présence d'une liaison étroite entre ces 2 loci : liaison de 7,5 unités pour Keller (1936), de 8,4 unités pour Owen et Ryser (1942) et de 6,3 unités pour Linde-Laursen (1972).

La contradiction entre ces 2 séries de résultats a été relevée par Bandlow (1955) qui pense que les loci $R$ doivent être différents chez les betteraves fourragères et les betteraves potagères.

La couleur des racines, les rapports mendéliens théoriques, les valeurs du $\chi^{2}$ et les probabilités correspondantes sont indiqués, pour les 6 générations du croisement $I$, dans le tableau VI. La disjonction observée en $F_{2}$, non différente d'une disjonction 9.3.3.1., indique que les loci $R$ et $Y$ sont indépendants (les $3 \chi^{2}$ partiels de la
$F_{2}$ : disjonction $\mathrm{Rr}, \mathrm{Yy}$ et leur test de linkage sont également non significatifs). Ces résultats sont donc conformes à ceux des premiers auteurs. La formule rrYY a été attribuée au parent $P_{1}$ et la formule RRyy au parent $P_{2}$.

$\mathrm{Si}$ nous nous reportons à la description des différents allèles $R$ et $Y$ donnée par Keller, Owen et Ryser, nous n'avons pas lieu de croire que l'allèle $R$ du parent $P_{2}$, qui provient de la souche USDA C9561, soit différent de l'allèle $R$ des variétés américaines de betterave sucrière, décrit dans le type I de Keller. Remarquons seulement que cet allèle $R$ betterave sucrière a ensuite été confondu par ces auteurs avec un allèle $R$ provenant d'une variété américaine de betterave potagère. $P$ ar contre, si l'allèle $Y d u$ parent $P_{1}$ présente bien, sous nos conditions, un hypocotyle jaune dans les conditions normales d'éclairement en plein champ, les limbes et les pétioles sont exempts ultérieurement de la coloration jaune signalée dans les types VIII et IX de Keller. Le facteur $Y$ de Keller provient de la même variété de betterave potagère que précédemment mais aussi, semble-t-il, d'une variété de betterave fourragère.

Nous avons donc tendance, pour notre part, à penser que l'allèle $Y$ de notre parent $P 1$ est différent de l'allèle $Y$ utilisé par les auteurs américains et il y aurait donc, apparemment au moins, 2 loci $R$ et 2 loci $Y$. L'existence de ces différents loci pourrait être démontrée à l'aide de tests d'allélisme pratiqués systématiquement sur les différents matériels d'étude encore disponibles actuellement. Ces tests seraient toutefois beaucoup facilités si l'on disposait, au préalable,

Tableau VI. Coloration des racines, rapports théoriques, test $\chi^{2}$ et probabilités correspondantes dans les 6 générations du croisement $I: P_{\dagger}(\mathrm{OLI}) \times \mathrm{P}_{2}(561-20)$.

Couleur des racines

\begin{tabular}{|c|c|c|c|c|c|c|c|}
\hline \multirow[t]{2}{*}{ Génération } & \multirow[t]{2}{*}{ Nombre de racines } & \multirow[t]{2}{*}{ Rouge } & \multirow[t]{2}{*}{ Orange } & Blanche à hypocotyle & \multirow[t]{2}{*}{ Rapport théorique } & \multirow[t]{2}{*}{$\chi^{2}$} & \multirow[t]{2}{*}{ Probabilité } \\
\hline & & & & Rouge * & & & \\
\hline
\end{tabular}

\begin{tabular}{|c|c|c|c|c|c|c|c|c|}
\hline$P_{1}$ & 398 & & 398 & & & & & \\
\hline$P_{2}$ & 398 & & & 398 & & & & \\
\hline$F_{1}$ & 414 & 414 & & & & & & \\
\hline$F_{2}$ & 402 & 222 & 79 & 79 & 22 & 9.3.3.1 & 0,81 & $0,80-0,90$ \\
\hline $\mathrm{B}_{1}$ & 367 & 198 & 169 & & & 1.1 & 2,45 & $0,10-0,20$ \\
\hline$B_{2}$ & 391 & 205 & & 186 & & 1.1 & 0,92 & $0,30-0,50$ \\
\hline
\end{tabular}

* La coloration rouge de l'hypocotyle se retrouve, à la récolte, dans la coloration de l'apex du bouquet foliaire des racines. 
de lignées fixées analogues à celles qui ont été utilisées dans le cadre de cette étude.

\section{DISCUSSION}

En reprenant successivement chacun des 3 caractères quantitatifs étudiés, on remarque que, dans les 2 croisements entre lignées de betterave fourragère et sucrière, l'expression du caractère poids frais des racines est déterminée en priorité soit par des effets de dominance (croisement I) soit par des effets d'épistasie (croisement II) et, secondairement, par des effets d'épistasie et d'additivité. Ces résultats sont conformes à ceux des travaux récents sur l'hérédité du caractère poids des racines de betterave sucrière, travaux qui ont montré que la plus grande partie de la variance génétique totale est due à une action non additive des gènes (Hecker, 1967; Smith et al, 1973; Johansson, 1984; Srivastava et al, 1986).

Pour la teneur en matière sèche, l'expression du caractère est déterminée, en priorité, par des effets d'additivité et, secondairement, par des effets d'épistasie (croisement I), de dominance et d'épistasie (croisement II) qui se traduisent par des valeurs d'hétérosis positives et très significatives dans les 2 croisements. Ces résultats sont à rapprocher des études sur l'hérédité de la teneur en sucre chez la betterave sucrière. Bien que la croyance soit encore répandue que cette teneur dépende seulement de l'action de gènes additifs, plusieurs publications anciennes et récentes (Peterson et Dickenson, 1958; Powers et al, 1959; Oldemeyer et Rush, 1960; Helmerick et al, 1963; Pylman, 1968; Srivastava et al, 1986) ont démontré l'existence d'une valeur d'hétérosis positive significative et cet hétérosis a été attribué également à des effets de dominance et d'épistasie (Johansson, 1984). En définitive, il ne semble pas que l'hérédité du caractère teneur en matière sèche chez la betterave soit fondamentalement différente de celle de la teneur en sucre (le sucre constitue, nous l'avons vu, les 2/3 environ du poids de la matière sèche). L'hérédité de la teneur en sucre a également été étudiée dans quelques croisements de variétés de betterave sucrière et fourragère. Ainsi Colin et Bougy (1939) puis Bougy (1942) constatent un accroissement important de la teneur en sucre de l'hybride par rapport à la moyenne des parents : 12,16 et 10,47 respectivement dans les croisements Kuhn $x$ Mangold, 15,45 et 12,13 dans le croisement Vilmorin $\times$ Vauriac, 11,11 et 9,80 dans le croisement Kuhn $\times$ Vauriac. En croisant une lignée mâle-stérile de betterave sucrière par la variété de betterave fourragère Ovana, Mir (1968) note un accroissement de la teneur en sucre de l'hybride plus modeste $(11,89$ et 11,22$)$, mais très significatif, et donc plus proche de nos propres observations. Mir cite également plusieurs croisements réalisés par Savitsky (1940) dans lesquels la teneur en sucre de l'hybride est égale à celle de la moyenne des 2 parents.

L'expression du caractère poids sec des racines, le plus important agronomiquement, est déterminée, en premier lieu, par des effets de dominance (croisement l) et par des effets d'épistasie (croisement II) et, secondairement, par des effets d'épistasie et d'additivité. La totalité ou la quasi-totalité de la variation génétique est due à une action non additive des gènes. À titre de comparaison avec le rendement en sucre pour la betterave sucrière, cette action non additive des gènes représente $52 \%$ de la variance génétique pour Srivastava et al, et $83 \%$ en présence d'un taux d'azote élevé dans le sol, $67 \%$ dans le cas contraire) pour Smith et al (1973).

Les 4 lignées utilisées dans les croisements 1 et II ont été choisies au hasard dans une collection de lignées fixées de betterave fourragère et sucrière. Leur valeur propre n'a pas d'intérêt particulier, leur valeur en combinaison est faible ou très faible et elles sont impropres à une utilisation dans les variétés commerciales mais elles présentent, toutes les 4 , un taux d'homozygotie très élevé. La présence de ces 2 caractères : choix au hasard et utilisation de lignées fixées permet de penser que les conclusions tirées de cette étude possèdent une certaine généralité. Elles devraient néanmoins être confirmẹées par des études analogues.

L'examen des résultats a mis en évidence l'importance des effets de dominance mais surtout l'importance, la fréquence et la diversité des effets d'interaction épistatique dans l'expression des 3 caractères quantitatifs étudiés. Ces effets d'interaction sont présents en des lieux et au cours d'années différents et ils contribuent, au moins autant que les effets de dominance, aux différentes manifestations de l'hétérosis. Johansson avait déjà montré la présence d'épistasie, pour différents caractères, dans les générations issues du croisement de certaines lignées monogermes sélectionnées de betterave sucrière. Les résultats ci-dessus indiquent que des effets d'épistasie importants existent également dans les croisements de lignées monogermes et multigermes non choisies et non sélectionnées au 
préalable et cela aussi bien en betterave fourragère qu'en betterave sucrière, c'est-à-dire dans un matériel considéré, respectivement, comme peu évolué et évolué génétiquement.

Ces résultats permettent également de proposer une hypothèse sur les bases génétiques de I'hétérosis dans les 2 croisements. En effet, si l'on admet, comme nous l'avons montré pour le caractère montée à graines (Le Cochec et Soreau, 1989a) que le mode d'action des gènes se simplifie progressivement en provoquant une diminution de l'hétérosis au cours de la vie de la plante, il est vraisemblable que les manifestations d'hétérosis constatées pour les 3 caractères sont, pour l'essentiel, le résultat d'effets d'épistasie au stade juvénile. Plus précisément, il s'agit sans doute d'effets d'épistasie trigénique ou d'ordre supérieur compliqués par des déviations dues à des linkages entre loci. Cette hypothèse n'est pas compatible avec la théorie de la dominance, théorie selon laquelle l'hétérosis serait la conséquence d'une dispersion de gènes dominants complémentaires chez les 2 parents. Elle diffère également de la théorie de la superdominance puisqu'elle amène à considérer l'hétérosis comme le résultat d'interactions complexes entre allèles situés sur un grand nombre de loci différents.

La présence d'effets d'épistasie et de superdominance dans les manifestations de l'hétérosis conduit à recommander la mise en œuvre d'un schéma de sélection récurrente réciproque entre les 2 origines betterave fourragère et betterave sucrière. C'est par le test systématique de la valeur en combinaison de lignées fixées de chaque origine que cet hétérosis pourrait être exploité au mieux et que la productivité des hybrides de betterave fourragère pourrait être améliorée, à la fois pour le poids frais et pour le poids sec des racines. Ces résultats constituent aussi un argument pour considérer que le test d'aptitude à la combinaison de lignées diploïdes bien définies est un moyen efficace d'amélioration de la teneur et de la productivité en sucre des hybrides actuels de betterave sucrière.

Nous avons eu l'occasion, à différentes reprises dans le courant de cet article, de montrer l'intérêt des lignées fixées pour l'étude des caractères génétiques et pour l'exploitation de la vigueur hybride chez la betterave. On peut rappeler que de telles lignées peuvent être obtenues, soit par l'utilisation de l'allèle d'autofertilité $S_{f}$ (c'est le cas de la plupart des lignées monogermes mâle-stériles), soit, comme nous l'avons fait (Le Cochec et Soreau, 1989b), par une sélection progressive pour l'aptitude à la pseudocom- patibilité, soit enfin, comme on le recherche maintenant, par un doublement chromosomique de lignées haploïdes obtenues par gynogénèse.

\section{RÉFÉRENCES}

Bandlow G (1955) Die Genetik der Beta vulgaris Rüben (Sammelreferat). Der Züchter 25, 104-122

Bougy E (1942) Dissociation d'hybrides de betteraves sucrières et fourragères. Public Inst Belge Amélior Bett 10, 35-43

Cockerham CC (1986) Modifications in estimating the number of genes for a quantitative character. Genetics 114, 659-664

Colin $H$, Bougy $E$ (1939) Les croisements de sucrières et de fourragères. Dissociation en $F_{2}$ de l'hybride Kuhn $\times$ Mangold. Publ Inst Belge Amélior Bett 7, 29-45

Demarquilly C (1972) Digestibilité, valeur nutritive et ingestibilité des betteraves de différentes teneurs en matière sèche. Ann Zootech 21, 3, 415-428

Hecker RJ (1967) Evaluation of three sugar beet breeding methods. J Am Soc Sugar Beet Technol 14, 309-318

Helmerick RH, Finkner RE, Doxtator GW (1963) Variety crosses in sugar beets (Beta vulgaris $\mathrm{L}$ ). I. Expression of heterosis and combining ability. $J A m$ Soc Sugar Beet Technol 12, 573-584

Jinks JL (1983) Biometrical genetics of heterosis. In: Heterosis. Springer-Verlag, Berlin, 1-46

Johansson E (1984) Estimation of genetic components of variation in sugar beets (Beta vulgaris $\mathrm{L}$ ) with special emphasis on epistatic interactions. Thèse Univ Genet Lund, $116 p$

Kajanus B (1917) Uber die Farbenvariation der BetaRüben. Z Pflanzenzuecht 5, 357-372

Keller W (1936) Inheritance of some major color types in beets. J Agric Res 52, 27-38

Lande R (1981) The minimum number of genes contributing to quantitative variation between and within populations. Genetics 99, 541-553

Le Cochec F, Soreau P (1989a) Mode d'action des gènes et hétérosis pour le caractère montée à graines dans le croisement de deux lignées fixées de betterave à sucre (Beta vulgaris L). Agronomie 9, 585-590

Le Cochec F, Soreau P (1989b) Évolution des lignées pseudocompatibles de betterave fourragère et sucrière (Beta vulgaris $L$ ) au cours des générations d'autofécondation. Agronomie 9, 1001-1011

Linde-Laursen IB (1972) A new locus for colour formation in beet, Beta vulgaris L. Hereditas 70, 105-112

Lindhard E, Iversen K (1920) Vererbung von roten und gelben Farbmerkmalen bei Beta-Rüben. Z Pflanzenzuecht 7, 1-18

Mac Farlane JS, Skoyen $1 O$ (1965) Sugar beet breeding lines combining resistance to bolting and disease. J Am Soc Sugar Beet Technol 13, 555-562 
Mather K, Jinks JL (1977) Introduction to biometrical genetics. Chapman and Hall, Londres, $231 \mathrm{p}$

Mir AR (1968) Gene analysis by the partitioning method of some crosses between sugarbeets and fodderbeets. Thèse, Colorado State Univ, $131 p$

Oldemeyer DL, Rush GE (1960) Evaluation of combining ability in self-fertile lines of sugar beets using male-sterile testers. J Am Soc Sugar Beet Technol $11,175-185$

Owen FV, Ryser GK (1942) Some Mendelian characters in Beta vulgaris $L$ and linkages observed in the Y-R-B group. J Agric Res 65, 3, 155-171

Peterson DF, Dickenson DD (1958) Results of divergent selection for general combining ability. $J \mathrm{Am}$ Soc Sugar Beet Technol 10, 60-65

Powers LR, Finkner RE, Rush GE, Wood RR, Peterson DF (1959) Genetic improvement of processing quality in sugar beets. $J$ Am Soc Sugar Beet Technol 10, 578-593
Pylman RW (1968) Inheritance of percent sucrose and weight of roots for crosses between three inbred sugarbeets. Thèse Colorado State Univ $109 p$

Savitsky VF (1940) A study of sucrose inheritance. Sueklovodstvo 1, 600-650

Smith GA, Hecker RJ, Maag GW, Rasmusson DM (1973) Combining ability and gene action estimates in an eight parent diallel cross of sugarbeet. Crop Sci 13, 312-316

Srivastava HM, Raman Kapur, Srivastava BL (1986) Heterosis, combining ability and gene action in a seven parent diallel in sugarbeet. Indian $J$ Genet $46,484-489$

Wright S (1968) Evolution and the genetics of populations. Vol I. Genetic and biometric foundations. Univ Chicago Press, Chicago, 469 p

Zeng ZB, Houle D, Cockerham CC (1990) How informative is Wright's estimator of the number of genes affecting a quantitative character? Genetics 126, 235-247 